\title{
Outcomes of Percutaneous Pinning Technique in the Management of Two-Part and Three-Part Proximal Humerus Fracture in Adults
}

Abdulkadr Muhammed Sleman Alany (MBChB,FIBMS) ${ }^{1}$, Hasan Mazin Shekheel Mercalose $(\mathrm{MBChB})^{2}$ and Thaqaf Alauldeen Fadhil Al-Bayati $(\mathrm{MBChB})^{3}$

\section{Abstract}

Background: Proximal humerus fractures represent $4-5 \%$ of over-all fractures. In patients above 40 years of age, it accounts for $75 \%$ of all humerus fractures. Fractures of the proximal end of the humerus oftentimes represent a management dilemma with the multiple surgical options available to deal with them.

Objective: To evaluate the functional and radiographic outcomes of the percutaneous pinning techniques in the management of two-part and three-part proximal humerus fracture. Patients and Methods: A case series of 25 patients, functional outcome was determined by ASES and Constant scores and multiple radiographic views were taken to assess the fracture union, mal-union, and the presence of avascular necrosis.

Results: ASES score of the whole group was 87.27 while the mean Constant score was 79.04. Only $16 \%$ of the entire group developed varus malunion (defined by Neck-Shaft-Angle $<=120$ degrees). Moreover, $16 \%$ of the whole group developed OA of the glenohumeral joint.

Conclusion: This study supports that the percutaneous close reduction and pinning technique is an effective and reliable surgical technique in the management of closed two-part and three-part proximal humerus fractures in adults. Appropriate preoperative planning is important to achieve a successful surgical result, and regular postoperative follow-up and rehabilitation are essential to get an optimum functional outcome.

Keywords: Proximal humerus fracture, percutaneous pinning, upper extremity surgery, trauma surgery, Clinical outcomes, Percutaneous fracture fixation

Corresponding Author: hasan.mercalose@gmail.com

Received: $17^{\text {th }}$ May 2020

Accepted: $19^{\text {th }}$ July 2020

DOI:https://doi.org/10.26505/DJM.19025380517

\footnotetext{
${ }^{1}$ College of Medicine- Hawler Medical University- Erbil- Iraq

${ }^{2,3}$ Erbil Teaching Hospital- Erbil- Iraq
}

\section{Introduction}

Proximal humerus fractures, defined as fractures occurring at or proximal to the surgical neck of the humerus, represent $4-5 \%$ of over-all fractures [1]. In patients above 40 years of age, it accounts for three quarters of all humerus fractures [2]. Fractures of the proximal end of the humerus oftentimes represent a management dilemma. Understanding the personality of each particular fracture may range from very 
straightforward to a quite complicated process [3].

The most commonly used classification system is that published by Neer. this classification system describes fractures as one, two, three or four-part fractures depending on the number of displaced segments, displacement is considered as being greater than one centimeter or more than $45^{\circ}$ angulation of a segment relative to another [4]. Displaced three-part and fourpart fractures markedly alter the articular congruity of the glenohumeral joint and have the highest likelihood of disrupting the major blood supply to the proximal humerus [5]. Surgical management of these fractures includeing: close reduction and percutaneous pinning, open reduction and internal fixation by multiple options including: plate and screws, fixed-angle device, transosseous suture fixation and locked intramedullary nail. The surgical technique of close reduction and percutaneous pinning was first described by Bohler [6] for the treatment of proximal humerus fracture in paediatric age group; however, this technique has been adopted for adult fractures as well. Percutaneous techniques reduce stripping and dissection around the shoulder joint, therefore, there is less risk of endangering the blood supply of the humeral head. Moreover, there is less scarring around the scapulohumeral joint thus the postoperative rehabilitation is easier.

The purpose of this study is to report the functional, clinical, and radiological outcomes of percutaneous pinning technique in two-part and three-part proximal humerus fractures in adults.

\section{Patients and Methods}

This case-series study is designed to report the outcomes of percutaneous pinning technique in two-part and three-parts proximal humerus fracture. Two emergency hospitals were involved in this study which are Balsam Private Hospital and West-Erbil Emergency Hospital in Erbil city. The operations were performed from January 2018 to February 2019. The data of this study were collected in the follow-up visit one year after the operations.

The inclusion criteria are adult patients (more than 18 years), two-part and three-part Neer fractures and the patients who had close fractures. While the exclusion criteria consist of: paediatric patients, proximal humerus fracture which has an extension to the shaft, pathological fractures, fractures with nerve and/or vascular injuries to the involved limb.

Informed consent was taken from all patients involved in this study and the research protocol had been accepted by the ethical committee of the Kurdistan Board of Medical Specialities which ensured the adherence of this study to the ethical principles.

\section{Surgical techniques}

After admission, all patients underwent a thorough clinical examination. The fracture was assessed by anteroposterior (AP) and axillary view (when possible). Moreover, a computed tomography scan (CT-Scan) was performed for all the cases. All these operations were done within one week after admission.

The decision of operation was made by a senior consultant, and the patients were informed about the operation, the possible 
outcomes and other alternative treatments. The operations were done under general anaesthesia in a semi-sitting (beach-chair position). An important step in patient positioning was that to put the involved scapula over the edge of the table to make an appropriate space for the fluoroscopy which was positioned to make orthogonal imaging of the proximal humerus possible and was used to guide fracture reduction and placement of percutaneous fixation. Proper patient positioning and ensuring that fluoroscopy images views are accepted, before the draping, is an essential step in this procedure.

Close reduction, as described by Jaberg et al [7], was an attempt in all the cases, and mini-invasive techniques using blunt instruments or a Steinmann-pin or K-wires were used to align the main parts aligned with the shaft.
To achieve the fixation, firstly two $2.5 \mathrm{~mm} \mathrm{~K}$ wires were passed through the lateral aspect of humeral shaft, above deltoid insertion (green lines), then another one is placed through the anterior cortex of the humeral shaft to the humeral head (the blue line); in case of greater tuberosity was fractured and displaced, then two pins were inserted antegrade from the greater tuberosity to the medial neck(red lines), sometimes a third Kwire was added to fix the greater tuberosity to the humeral head. In addition, more Kwires were added to achieve a more stable fixation if the fracture configuration or the bone quality requires more stability. The Kwires were carefully bent and cut $2 \mathrm{~cm}$ outside the skin and proper dressing was applied (Figure 1) and (Figure 2). The arm was held in an arm-sling. and an abduction pillow was used in all the patients to keep the arm in an abducted valgus position.

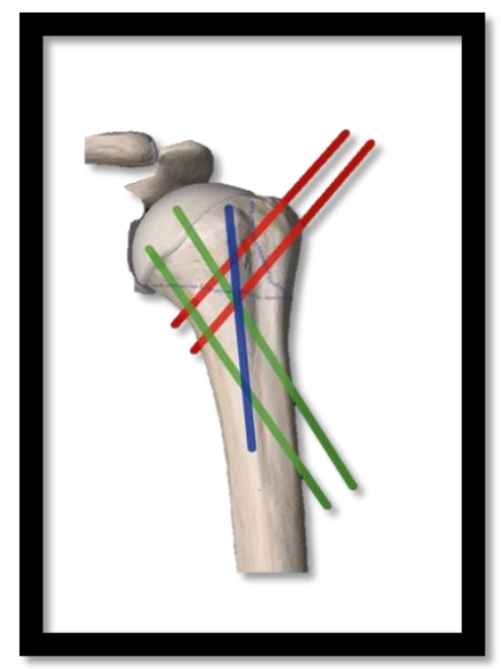

Figure (1): The techniques of percutaneous fixation

\section{Postoperative care and rehabilitation}

From the first postoperative day the patients were encouraged to start active exercises of the elbow, forearm, wrist and hand to avoid stiffness of these joints.
Meanwhile, the shoulder joint was immobilized (in an arm-sling and abduction pillow) for 4 weeks when the first radiograph was taken and loose wires were removed (if present). Moreover, the K-wires were 
removed between week 6 and 8 postoperatively. At the sixth week, passive and active exercises were started and the strength was increased gradually. Thereafter, these patients were assessed in the third and
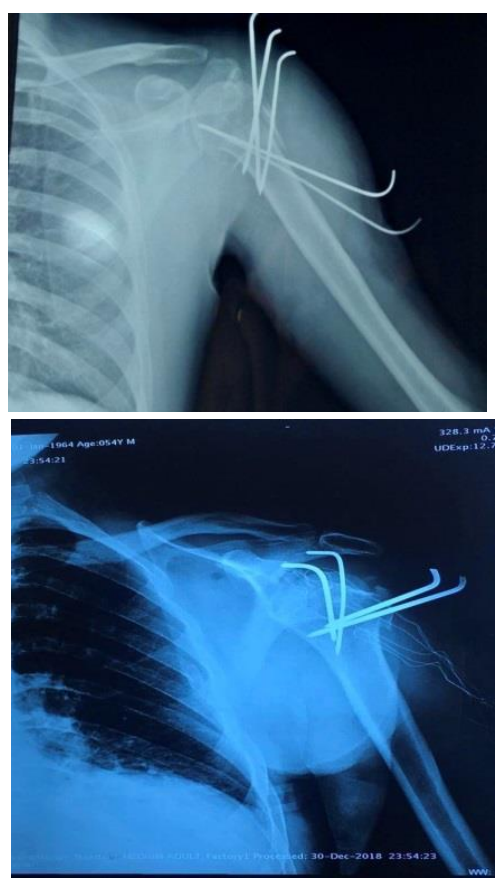

Figure (2): Postoperative images using percutaneous reduction technique and fixation by K-wires

\section{Evaluation after one year}

At a minimum of one year after surgery, the follow-up assessment for this study was performed. All patients filled questionnaire forms regarding their shoulder joint function and pain. A standard goniometer was used to assess the active and passive range of movement at the shoulder joint. For pain evaluation, the pain numeric scale, ranging from 0 (no pain) to 10 (the worst pain), was used. Rotator cuff muscles strength were measured manually, and the power of shoulder abduction was quantified with an electronic dynamometer. The functional outcome of the shoulder was determined by calculating the American Shoulder and Elbow Surgeons (ASES) [8] score and the Constant score [9] and for each patient. sixth months after the operation. At one year postoperatively, we assessed each patient for the measures that will be mentioned in the later lines.

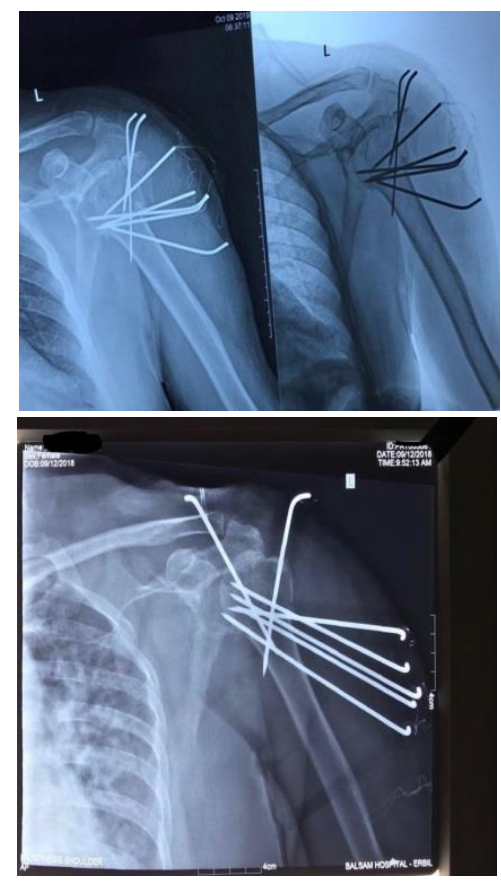

Radiographs were taken at the annual follow-up including anteroposterior (AP), true AP, and lateral axillary views, each radiograph was assessed for signs of avascular necrosis, mal-union and osteoarthritis (OA) of the glenohumeral joint. Avascular necrosis was determined by the presence of a discrete area of sclerosis or collapse in the humeral head. Osteoarthrosis was classified to be grade-one if the joint space was narrowed, grade-two if there were osteophytes of less than $3 \mathrm{~mm}$, grade-three if significant subchondral sclerosis was present in addition to osteophytes, and grade-four in the presence of greater changes [10]. Moreover, Neck-shaft-angle (NSA) on true AP view was used to determine the presence of malunion which was defined by a Neck- 
shaft angle less than 120 or more than 160 degrees. The NSA was calculated by measuring the angle between the intersection of a line along the axis of the proximal humerus and another line which is perpendicular to the anatomic neck of the humerus.

\section{Statistical analysis}

Data were analyzed using the Statistical Package for Social Sciences (SPSS, version 25). Chi-square test of association was used to compare proportions. Fisher's exact test was used when the expected count of more than $20 \%$ of the cells of the table was less than 5. Mann Whitney test was used to compare the mean ranks of the parameters of the two groups (two and three parts fractures). A p-value of $\leq 0.05$ was considered statistically significant.

\section{Results}

Twenty-five patients with fracture humerus were included in the study. The highest proportion of the patients $(44 \%)$ were aged 55-64 years old, and more than half $(60 \%)$ were females. The majority $(76 \%)$ of the patients were right-handed, and the involved side was the right side in $60 \%$ of the patients. Regarding the type of the fracture, it was a two-part fracture in $52 \%$ of the patients, and a three-part fracture in $48 \%$ of the cases.

Osteoarthritis developed in $16 \%$ of the patients. None of the patients developed nonunion or avascular necrosis. Regarding the mean $( \pm$ SD) neck-shaft angle, it was 132.04 $\pm 10.79^{0}$ Table (2).

\section{Physical Examination}

On examination, the majority of the patients achieved either thoraco-lumber $(40 \%)$ or thoracic $(44 \%)$ internal rotation, but there was no significant $(\mathrm{p}=0.191)$ difference between the patients with two and three parts fractures as presented in Table 4. A round half $(48 \%)$ of the patients achieved $160-180^{\circ}$ forward flexion but the difference between the two groups was not significant $(\mathrm{p}=0.158)$. Regarding the external rotation, $76 \%$ of the patients were normal $(92.3 \%$ in the two parts fractures and $58.3 \%$ in the three parts fractures), but the difference was not significant $(p=0.073)$. The mean numerical pain score of the entire group was 1.04/10 with more than half $(56 \%)$ of the patients had only mild pain, scores less than or equal to $3 / 10$, (46.2\% in the two parts fractures and $66.7 \%$ in the three parts fractures), again the difference was not significant $(p=0.302)$. none of the patients had a pain sore more than $3 / 10$. The parameters of the functional outcomes are presented in Table (3).

\section{Outcomes Evaluation}

The mean ASES score of the whole group was $87.27( \pm 3.39)$ while the mean rank of the ASES score of the two-part fracture was significantly higher than the mean rank of the three-parts fracture $(\mathrm{p}=0.001)$. The Constant score of the whole group was $79.04( \pm 5.12)$ and it was higher in the two-part group but the difference with the three-part was not significant $(\mathrm{p}=0.913)$ as presented in Table (5). 


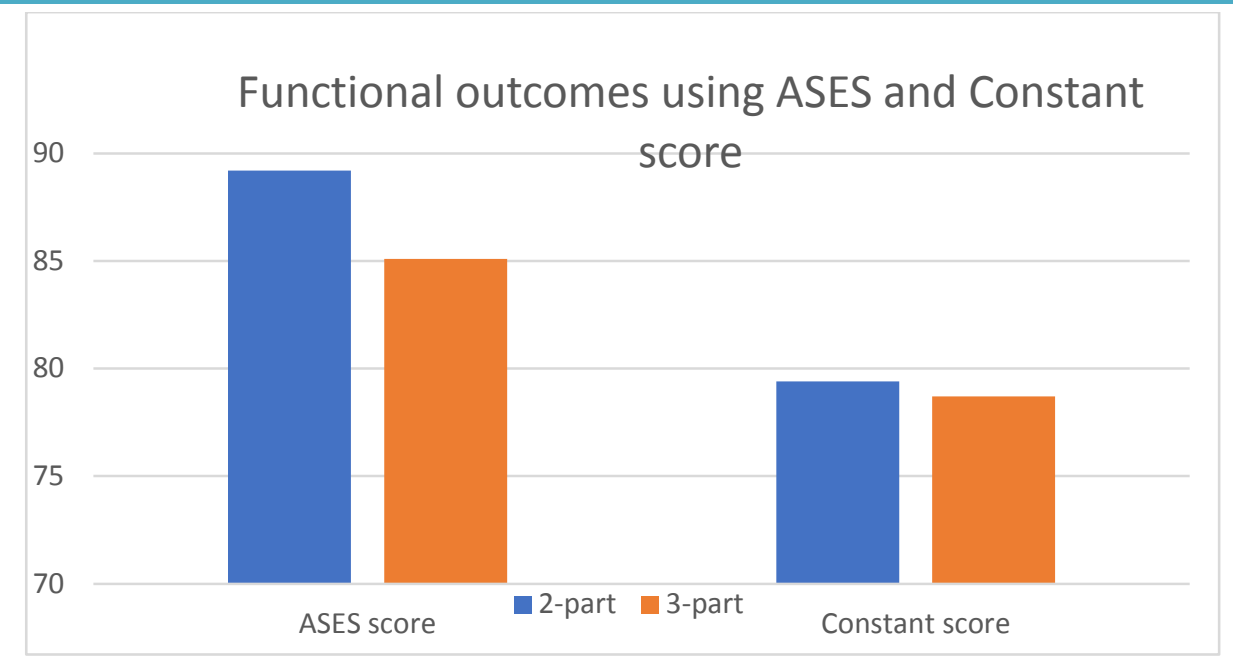

Figure (3): Functional outcomes according to the fracture

The ASES score was significantly higher among those with no osteoarthritis (OA) than patients with OA $(\mathrm{p}=0.002)$. The same pattern was noticed with the Constant Score but the difference was not significant $(\mathrm{p}=$ 0.352). The pain was significantly higher among patients with OA compared with patients who didn't develop OA $(\mathrm{p}=0.001)$ as presented in Table (6). Table (7) shows that $33.3 \%$ of patients with three parts fracture developed OA in the glenohumeral joint in the form of irregularity and narrowing of the joint space, while none of the patients with two parts fracture developed OA $(\mathrm{p}=0.039)$.

Only $16 \%$ of the patients developed malunion (Neck-shaft-angle below 120 degrees), but the difference was not significant between the two groups ( $p$ $>0.999$ ). When comparing the parameters of the functional outcome (ASES score, constant score, and pain scores) between patients with mal-union and patients with union, no significant differences were detected $(\mathrm{p}=0.765, \mathrm{p}=0.823$, and $\mathrm{p}=0.610$ respectively) as presented in Table (8).

\section{Complications}

Regarding postoperative complications, none of the patients developed nerve or vascular injuries, however, pin-loosening was observed in one patient that required pin removal 25 days after the operation. Moreover, one patient developed a pin-tract infection, this presented as a slight amount of discharge around one k-wire which did not affect the stability of the fracture and was treated by removal of the K-wire, wound care, and antibiotics guided by culture and sensitivity. In addition, no pin-migration or deep infections happened to any of these patients. 
Table (1): Basic characteristics of the studied sample

\begin{tabular}{||l||c||c||}
\hline \multicolumn{1}{|l||}{} & No. & (\%) \\
\hline \hline Age (years) & & $(24.0)$ \\
\hline \hline 555 & 6 & $(44.0)$ \\
\hline$\geq 5-64$ & 11 & $(32.0)$ \\
\hline \hline Gender & & \\
\hline \hline Male & 10 & $(40.0)$ \\
\hline \hline Female & 15 & $(60.0)$ \\
\hline Dominant side & & \\
\hline \hline Right & 19 & $(76.0)$ \\
\hline Left & 6 & $(24.0)$ \\
\hline \hline Involved side & & \\
\hline \hline Right & 15 & $(60.0)$ \\
\hline \hline Left & 10 & $(40.0)$ \\
\hline \hline Fracture type & & $(52.0)$ \\
\hline \hline Two parts & 13 & $(48.0)$ \\
\hline \hline Three parts & 12 & $(100.0)$ \\
\hline \hline Total & 25 & \\
\hline
\end{tabular}

Table (2): Radiographic outcomes

\begin{tabular}{|l|c|c|}
\hline & No. & (\%) \\
\hline \hline $\begin{array}{l}\text { Radiographic } \\
\text { evaluation }\end{array}$ & & \\
\hline \hline No osteoarthritis & 21 & $(84.0)$ \\
\hline \hline Osteoarthritis & 4 & $(16.0)$ \\
\hline \hline Fracture union & & $(100.0)$ \\
\hline \hline Yes & 25 & $(0.0)$ \\
\hline \hline No & 0 & $(0.0)$ \\
\hline \hline Avascular necrosis & & $(100.0)$ \\
\hline \hline Yes & 0 & \\
\hline No & 25 & $( \pm 10.79)$ \\
\hline \hline Neck shaft angle & & 132.04 \\
\hline Mean $( \pm$ SD $)$ & & \\
\hline \hline
\end{tabular}

Table (3): Physical examination and functional outcomes of the entire group

\begin{tabular}{|l|r|l|c|c|c||}
\hline & Mean & $( \pm$ SD) & Median & Min. & Max. \\
\hline \hline Forward flexion & 155.60 & $( \pm 13.33)$ & 155.00 & 130.00 & 175.00 \\
\hline \hline External rotation & 58.60 & $( \pm 12.12)$ & 60.00 & 40.00 & 80.00 \\
\hline \hline Abduction & 162.80 & $( \pm 10.01)$ & 165.00 & 145.00 & 180.00 \\
\hline \hline Pain (numerical rating scale) & 1.04 & $( \pm 1.14)$ & 1.00 & 0.00 & 3.00 \\
\hline \hline ASES score & 87.24 & $( \pm 3.39)$ & 87.00 & 83.00 & 97.00 \\
\hline \hline Constant score & 79.04 & $( \pm 5.12)$ & 80.00 & 71.00 & 95.00 \\
\hline
\end{tabular}


Table (4): Physical examination by type of fracture

\begin{tabular}{|c|c|c|c|c|c|c|c|}
\hline & \multicolumn{2}{|c|}{ Two parts } & \multicolumn{2}{|c|}{ Three parts } & \multicolumn{2}{|c|}{ Total } & \multirow[b]{2}{*}{$\mathbf{p}$} \\
\hline & No. & $(\%)$ & No. & $(\%)$ & No. & $(\%)$ & \\
\hline \multicolumn{8}{|l|}{ Internal rotation } \\
\hline Thoracic & 8 & (61.5) & 2 & $(25.0)$ & 11 & $(44.0)$ & \\
\hline Thoraco-lumber & 4 & $(30.8)$ & 6 & $(50.0)$ & 10 & $(40.0)$ & \\
\hline Mid-lumber & 1 & 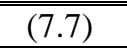 & 1 & (8.3) & 2 & (8.0) & \\
\hline $\begin{array}{l}\text { Gluteal } \\
\end{array}$ & 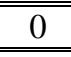 & 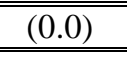 & 2 & (16.7) & 2 & $\overline{~(8.0)}$ & 0.191 \\
\hline \multicolumn{8}{|l|}{ Forward flexion } \\
\hline$<160^{\circ}$ & 5 & $(38.5)$ & 8 & (66.7) & 13 & (52.0) & \\
\hline $160-180^{\circ}$ & 8 & (61.5) & 4 & (33.3) & 12 & (48.0) & 0.158 \\
\hline \multicolumn{8}{|l|}{ External rotation } \\
\hline$<45^{\circ}$ & 0 & (20.0) & 2 & (16.7) & 2 & (8.0) & \\
\hline $45-90^{0}$ & 13 & $(100.0)$ & 10 & (83.3) & 23 & $\begin{array}{l}(92.0) \\
\end{array}$ & 0.220* \\
\hline \multicolumn{8}{|l|}{ Abduction } \\
\hline$<160$ & 1 & $\begin{array}{l}(7.7) \\
\end{array}$ & 5 & $(41.7)$ & 6 & $(24.0)$ & \\
\hline Normal & 12 & $(92.3)$ & 7 & $(58.3)$ & 19 & $(76.0)$ & 0.073 \\
\hline \multicolumn{8}{|l|}{ Pain } \\
\hline No pain & 7 & $(53.8)$ & 4 & (33.3) & 11 & $(44.0)$ & \\
\hline Mild & 6 & $(46.2)$ & 8 & $(66.7)$ & 14 & $(56.0)$ & 0.302 \\
\hline Total & 13 & (100.0) & 12 & $(100.0)$ & 25 & $(100.0)$ & \\
\hline
\end{tabular}

Table (5): Functional outcome as assessed by the ASES score and constant scores

\begin{tabular}{|c|c|c|c|c|c|c|c|}
\hline & Mean & $\overline{( \pm \mathrm{SD})}$ & Median & Minimum & Maximum & $\begin{array}{c}\text { mean } \\
\text { rank }\end{array}$ & $\overline{\mathbf{P}^{*}}$ \\
\hline \multicolumn{8}{|c|}{ ASES score } \\
\hline Two parts & 89.2 & $( \pm 3.3)$ & 88.0 & 85.0 & 97.0 & 17.7 & \\
\hline $\begin{array}{l}\text { Three } \\
\text { parts }\end{array}$ & 85.1 & $( \pm 1.8)$ & 84.5 & 83.0 & 88.0 & 7.9 & 0.001 \\
\hline \multicolumn{8}{|c|}{ Constant score } \\
\hline Two parts & 79.4 & $( \pm 6.4)$ & 80.0 & $\overline{771.0}$ & 95.0 & 13.15 & \\
\hline $\begin{array}{l}\text { Three } \\
\text { parts }\end{array}$ & 78.7 & $( \pm 3.6)$ & 79.0 & 73.0 & 85.0 & 12.83 & 0.913 \\
\hline
\end{tabular}

Table (6): Effect of osteoarthritis on the outcome

\begin{tabular}{|c|c|c|c|c|c|c|c|}
\hline & Mean & Median & ( $( \pm$ SD $)$ & Min. & Max. & $\begin{array}{c}\text { Mean } \\
\text { rank }\end{array}$ & $\mathbf{P}^{*}$ \\
\hline \multicolumn{8}{|l|}{ ASES score } \\
\hline No osteoarthritis & 88.00 & 8787.00 & 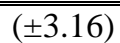 & 84.00 & 97.00 & 14.95 & 0.002 \\
\hline Osteoarthritis & 83.25 & 83.00 & $( \pm 0.50)$ & 83.00 & 84.00 & 2.75 & \\
\hline \multicolumn{8}{|l|}{ Constant score } \\
\hline No osteoarthritis & 79.48 & 80.00 & $\overline{( \pm( \pm 5.30)}$ & 71.00 & 95.00 & 13.60 & 0.352 \\
\hline Osteoarthritis & 76.75 & 76.00 & $( \pm 3.77)$ & 73.00 & 82.00 & 9.88 & \\
\hline \multicolumn{8}{|l|}{ Pain } \\
\hline No osteoarthritis & 0.67 & 0.00 & $( \pm 0.80)$ & 0.00 & 2.00 & 11.00 & 0.001 \\
\hline "Osteoarthritis & 3.00 & 3.00 & $( \pm 0.00)$ & 3.00 & 3.00 & 23.50 & \\
\hline
\end{tabular}


Table (7): Radiographic outcome by type of fracture

\begin{tabular}{|c|c|c|c|c|c|c|c|}
\hline & \multicolumn{2}{|c|}{ Two parts } & \multicolumn{2}{|c|}{ Three parts } & \multicolumn{2}{|c|}{ Total } & \multirow[b]{2}{*}{$\mathbf{p}$} \\
\hline & No. & $(\%)$ & No. & $(\%)$ & No. & $(\%)$ & \\
\hline \multicolumn{8}{|c|}{ Radiographic evaluation } \\
\hline No osteoarthritis & 13 & (100.0) & 8 & $3(66.7)$ & 21 & $3(84.0)$ & \\
\hline Osteoarthritis & 0 & $\begin{array}{l}(0.0) \\
\end{array}$ & 4 & (33.3) & 4 & (16.0) & $0.039 *$ \\
\hline \multicolumn{8}{|l|}{ Mal-union } \\
\hline Mal-union & 2 & (15.4) & 2 & $\begin{array}{l}(16.7) \\
\end{array}$ & $\overline{44}$ & $\begin{array}{l}(16.0) \\
\end{array}$ & \\
\hline Union & 11 & $\begin{array}{l}(84.6) \\
\end{array}$ & 10 & (83.3) & 21 & (84.0) & $>0.999 *$ \\
\hline Total & 13 & 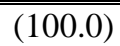 & 12 & 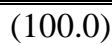 & 25 & $\overline{(100.0)}$ & \\
\hline
\end{tabular}

Table (8): Effect of malunion on functional outcome

\begin{tabular}{|l||c||c||c||c||c||c||c||}
\hline \hline & Mean & Median & $( \pm$ SD) & Min. & Max. & $\begin{array}{c}\text { Mean } \\
\text { rank }\end{array}$ & P* \\
\hline \hline ASES score & & & & & & & \\
\hline \hline Mal-union & 86.75 & 86.00 & $( \pm 3.10)$ & 84.00 & 91.00 & 12.00 & \\
\hline \hline Union & 87.33 & 87.00 & $( \pm 3.51)$ & 83.00 & 97.00 & 13.19 & 0.765 \\
\hline Constant score & \multicolumn{2}{|l|}{} & & & & \\
\hline Mal-union & 78.75 & 79.50 & $( \pm 4.65)$ & 73.00 & 83.00 & 13.75 & \\
\hline \hline Union & 79.10 & 80.00 & $( \pm 5.31)$ & 71.00 & 95.00 & 12.86 & 0.823 \\
\hline \hline Pain & & & & & & & \\
\hline \hline Mal-union & 1.25 & 1.00 & $( \pm 1.26)$ & 0.00 & 3.00 & 14.63 & \\
\hline \hline Union & 1.00 & 1.00 & $( \pm 1.14)$ & 0.00 & 3.00 & 12.69 & 0.610 \\
\hline
\end{tabular}

\section{Discussion}

Percutaneous reduction and pinning techniques lead to outstanding results and low complication rates in the management of two-part and three-part proximal humerus fracture. The advantages of this technique include less soft-tissue dissection, reliable healing, better cosmetic outcome and lower costs than open techniques due to relatively cheap instruments. These techniques require an accurate understanding of the fracture anatomy, and the deforming forces created by the muscles around the shoulder joint. The surgical skills of this procedure have a significant learning curve.

This study shows that most of the patients have returned to nearly normal functional level with least pain scores. The ASES score was significantly higher in the two-part group. The advantages of this technique include less soft-tissue dissection, a reliable healing, better cosmetic outcomes, and lower costs than open techniques due to relatively cheap instruments.

In this study, none of the cases developed avascular necrosis or non-union, while only $14 \%$ of the cases (all from the three-part group) developed osteoarthritis in the glenohumeral joint, this can be explained by almost anatomic reduction can be achieved by percutaneous techniques while decreasing the vascular compromise of the head of the humerus. Moreover, $16 \%$ of the sample developed a varus mal-union with statistically non-significant effect on the functional scores. 
Many researchers have reported the outcomes of minimally invasive techniques to the management of the proximal humerus fracture. Soete et al presented the outcomes in a case series of 31 patients who had undergone percutaneous reduction and fixation of proximal humerus fractures, and good results were presented [11]. Jay D. Keener et al reported the outcomes after percutaneous reduction and fixation of proximal humeral fractures, the mean Constant score of the two-part and there-part groups were 78.7 and 78.6 respectively while the ASES score of the two-part was 87.9 and for the three-part group was 85.1. Moreover, osteoarthritis developed in $17 \%$ of the cases and no avascular necrosis developed in the two-part and three-part fracture groups [12]. Jaberg et al presented a case series of 48 patients with different types of proximal humerus fractures which were reduced and fixed percutaneously, they reported excellent or good results by using the Salliant scale after three years of follow-up with only a $4 \%$ rate of avascular necrosis in the humeral head [7]. In a case series of 27 patients who were above the age of fifty years, Eid et al [13] described a percutaneous technique in these types of fractures based on using Shanz screws in combination with K-wires, the Constant Score of the two-part and three-part groups were 71.9 and 65.5 respectively, while the pin-tract infection was the most common complication. Comparing with other methods of fixation, Jaura et al [14] compared the functional outcomes using Constant and VAS scores in thirty patients who had undergone fixation by Proximal Humerus Internal Locking System (PHILOS) versus another 30 patients who were treated by percutaneous K-wire fixation, the mean Constant Score in the first group was 84.6 points while in the K-wire group was 76.4 , he concluded that internal fixation using PHILOS plate enabled the patients to start physiotherapy sooner, however, the functional results of both groups were acceptable.

We believe that percutaneous reduction and fixation techniques require an accurate understanding of the fracture anatomy and the deforming forces created by the muscles around the shoulder joint. The surgical skills of this procedure have a significant learning curve. Therefore, we believe that preoperative Computed Tomography (CT scan) is of paramount importance in this setting to get a proper assessment of the fracture type and to detect areas of comminution. Moreover, regular follow-up and ensuring appropriate physiotherapy during the rehabilitation period is essential to achieve a desired functional outcome.

Regarding the limitation in this study, a longer duration of follow-up could have revealed a better assessment of the long-term complications. In addition, a larger sample size would have demonstrated a better evaluation of these techniques against other variables like age, gender, and type of fracture.

\section{Conclusions}

Percutaneous close reduction and pinning is a reliable surgical technique in the management of closed two-part and threepart proximal humerus fractures in adults. Appropriate preoperative planning is important to achieve a successful surgical 
result, and regular postoperative follow-up and rehabilitation are essential to get an optimum functional outcome.

\section{Recommendations}

We suggest further research work in this field in prospective comparative studies comparing methods of open and close techniques in a larger sample, at multi-center levels, and for a longer duration of follow-up.

\section{References}

[1]Lind T, Kroner K, Jensen J. The epidemiology of fractures of the proximal humerus. Arch Orthop Trauma Surg 1989;108:285-7.

[2]Rose SH, Melton LJ III, Morrey BF, Ilstrup DM, Riggs BL. Epidemiologic features of humeral fractures. Clin Orthop Relat Res 1982: 24-30.

[3] Rockwood and Green Fractures in Adult 9th Edition, P. 1865.

[4]Neer CS 2nd. Displaced proximal humeral fractures. I: classification and evaluation. J Bone Joint Surg [Am] 1970;52-A:1077-89.

[5] Campbells's Operative Orthopedics, 13th Edition,Part Xv Fractures And Dislocations In Adults, Page 2936.

[6]Bohler J. Les fractures recentes de l'epaule. Acta Orthop Belg. 1964;30:235-42. [7]Jaberg H, Warner JJ, Jakob RP. Percutaneous stabilization of unstable fractures of the humerus. J Bone Joint Surg Am 1992; 74:508-15.

[8]Richards RR, An KN, Bigliani LU, Friedman RJ, Gartsman GM, Gristina AG, Iannotti JP, Mow VC, Sidles JA, Zuckerman JD. A standardized method for the assessment of shoulder function. J Shoulder Elbow Surg. 1994;3:347-352.
[9]Constant CR, Murley AH. A clinical method of functional assessment of the shoulder. Clin Orthop Relat Res 1987:160-4. [10]Gerber C, Hersche O, Berberat C. The clinical relevance of posttraumatic avascular necrosis of the humeral head. J Shoulder Elbow Surg 1998;7:586-90.

[11]Soete PJ, Clayson PE, Costenoble VH. Transitory percutaneous pinning in fractures of the proximal humerus. J Shoulder Elbow Surg 1999;8:569-73.

[12]Keener, J. D., Parsons, B. O., Flatow, E. L., Rogers, K., Williams, G. R., \& Galatz, L. M. (2007). Outcomes after percutaneous reduction and fixation of proximal humeral fractures. Journal of Shoulder and Elbow Surgery, 16(3), 330-338. https://doi.org/10.1016/j.jse.2006.09.006

[13]Eid A, Osman M, Fekry HE. Percutaneous fixation with Schanz screws for displaced two- and three- part fractures of the proximal humerus in patients above fifty years of age. Int $J$ Shoulder Surg. 2011;5(2):38-43. doi:10.4103/09736042.83195 .

[14]Jaura G, Sikdar J, Singh S. Long Term Results of PHILOS Plating and Percutaneous K-Wire Fixation in Proximal Humerus Fractures in The Elderly. Malays Orthop J. 2014;8(1):4-7. doi:10.5704/MOJ.1403.010 